\title{
Análisis comparativo del test de Bosco con técnicas de video en 3D (Peak Performance)
}

\author{
RUDOLF MORGENSTERN* \\ J. PORTA** \\ J.L. PARREÑO*** \\ D. RUANO GIL****
}

\begin{abstract}
* Director del Laboratorio de Biomecánica de la Escuela de Medicina de la Educación Física y el Deporte,
Profesor de la Facultad de Medicina, Universidad de Barcelona.

**Profesor del INEF de Barcelona.

***Licenciado en Educación Física, Doctorando del Departamento de Anatomía, Facultad de Medicina, Universidad de Barcelona.

**** Catedrático de Anatomía, Director de la Escuela de Medicina de la Educación Física y el Deprote, Facultad de Medicina, Universidad de Barcelona.
\end{abstract}

\section{INTRODUCCION}

El salto vertical constituye uno de los gestos más comúnmente utilizados en el deporte. Existen numerosos métodos para la valoración del mismo. En 1.983 Bosco presentó un método simple para la medida del tiempo de vuelo de un salto vertical ${ }^{1,2,3,4,5,6}$ y éste fue entonces usado para el cálculo del incremento de altura del centro de gravedad. Partiendo de las leyes de Newton la velocidad de caída sería:

$$
\mathrm{V}=\mathrm{Vo}+\mathrm{at}=\mathrm{Vo}+\mathrm{gt}=(2 \mathrm{gh})^{1 / 2}
$$

Donde $\mathrm{V}=$ Velocidad vertical, $\mathrm{Vo}=$ Velocidad inicial, $\mathrm{a}=$ aceleración, $\mathrm{h}=$ altura, $\mathrm{t}=$ tiempo de vuelo $\mathrm{y}$ $\mathrm{g}=\mathrm{a}$ celeración debida a la gravedad.

Haciendo Vo $=0$ en el punto máximo y sustituyendo en 1:

$$
\mathrm{V}=0+\mathrm{gt}=(2 \mathrm{gh})^{1 / 2}
$$

Haciendo $T=t / 2$, siendo $T$ el tiempo de máxima elevación y despejando $\mathrm{h}$ :

$$
\mathrm{h}=\frac{\mathrm{gT}^{2}}{8}
$$

Este estudio pretende comparar el test de Bosco realizado con el tapiz de contacto llamado «Ergojump»
(Junghans GMBH-Schramberg, BRD) con la trayectoria del centro de gravedad (CG) del sujeto filmada en video, digitalizada y computada en tres dimensiones. Para ello se utilizará un sistema de análisis del movimiento en tres dimensiones capaz de medir el desplazamiento estrictamente vertical de CG versus el «Ergojump» constituido por un «timer» $( \pm 0,0001 \mathrm{~s})$ conectado por un cable a una plataforma. El «timer» se pone en marcha cuando el pie del sujeto deja de contactar con la plataforma y se para en el momento de la toma de contacto.

\section{MATERIAL Y MÉTODOS}

Se dispuso de una población de 32 atletas con la siguiente distribución por deportes: 12 triathletas, 8 ciclistas, 4 culturistas y 2 corredores de motocros y de 6 sedentarios. Las medidas de altura, edad y peso se muestran en la tabla 1.

Los sujetos fueron instruidos en la realización de dos tipos de salto sobre una plataforma de Bosco:

1) Squatting Jump (SJ): partiendo el sujeto de una posición de semisquat sin realizar un contra-movimiento preparatorio, en dicha posición el ángulo de la rodilla se encuentra aproximadamente situado a $90^{\circ}$.

2) Counter-Movement Jump (CMJ): El sujeto parte de una posición erecta realizando posteriormente un con-

TABLA I

\begin{tabular}{lrrrrr}
\hline & SEDENTARIOS & TRIATHLON & CICLISMO & CULTURISMO & MOTORISMO \\
\hline EDAD (años) & 23.11 & 26.90 & 28.00 & 30.50 & 26.00 \\
TALLA (cm) & 167.21 & 170.30 & 177.32 & 167.05 & 175.67 \\
PESO $(\mathrm{Kg}$.) & 56.53 & 64.29 & 68.87 & 75.62 & 66.12 \\
\hline
\end{tabular}


tra-movimiento preparatorio para el salto, el final de la fase de contra-movimiento se corresponde con la posición inicial del SJ.

En ambos saltos se debe evitar el desplazamiento lateral y horizontal, así como la participación de otra musculatura que no sea la extensora de la extremidad inferior, para ello el sujeto permaneció con las extremidades superiores cruzadas en la parte anterior del tórax e inmovilizadas por ligaduras.

Se marcó al individuo con bolas de poliestireno expandido recubiertas de material reflectante (al ser de este material se minimiza la masa y sus movimientos inerciales) en los siguientes puntos:

- Hombro derecho

- Hombro izquierdo

- Cadera derecha

- Cadera izquierda

- Rodilla derecha

- Rodilla izquierda

- Tobillo derecho

- Tobillo izquierdo

- Pie derecho

- Pie izquierdo

Los saltos sobre el tapiz fueron filmados con cámaras de alta resolución (Panasonic AG 450) en sistema $S$ VHS dispuestas como muestra la figura 1.

Posteriormente se procedió a la digitalización automática, para lo cual se creó un modelo biomecánico que permitió la digitalización del cuerpo en movimiento, determinando la situación instantánea del centro de gravedad. Se consideró cabeza, tronco y extremidades superiores como un bloque (fig. 2), tomando hombro derecho, hombro izquierdo, cadera derecha y cadera izquierda como límites y se calculó el centro de gravedad de dicho bloque. En las extremidades inferiores se consideraron los siguientes bloques: muslo derecho, muslo izquierdo, pierna dercha, pierna izquierda, pie derecho y pie izquierdo (fig. 3). Los datos se procesaron mediante un sistema informático (software Peak Performance procesado con un ordenador Compaq 386:20 MHz).
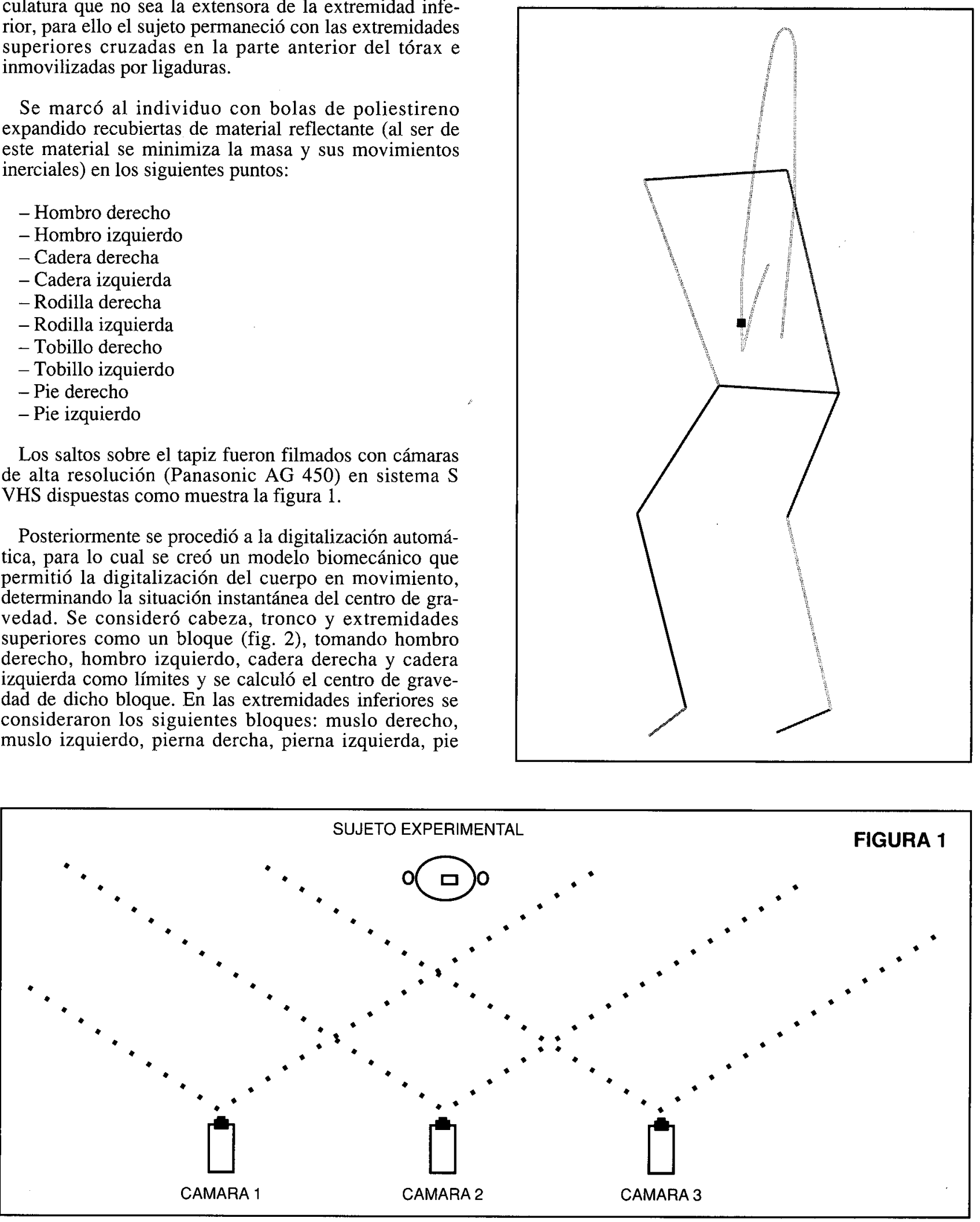


\section{FIGURA 3 \\ - CENTER OF MESS SET UP-}
1. L. ANKLE
4. L. SHOULDER
7. R. KNEE
2. L. KNEE
6. R. HIP
8. R. ANKLE
3. L. HIP
7. R. KNEE
9. R. FOOT

10. L. FOOT

LABEL P $D$ \%Dist. \%Mass Label

\begin{tabular}{|c|c|c|c|c|c|}
\hline L. THIGH & 3 & 2 & 37 & 10.3 & UP. BODY \\
\hline L. SHANK & 2 & 1 & 37 & 4.3 & \\
\hline R. THIGH & 6 & 7 & 37 & 10.3 & \\
\hline R. SHANK & 7 & 8 & 37 & 4.3 & - \\
\hline L. FOOT & 1 & 10 & 45 & 1.5 & \\
\hline R. FOOT & 8 & 9 & 45 & 1.5 & \\
\hline
\end{tabular}

$\begin{array}{lllll}P 1 & P 2 & D 1 & D 2 & \% \text { Dits }\end{array}$

$\%$ Mass

Position with cursor keys, enter $L \rightarrow R$.
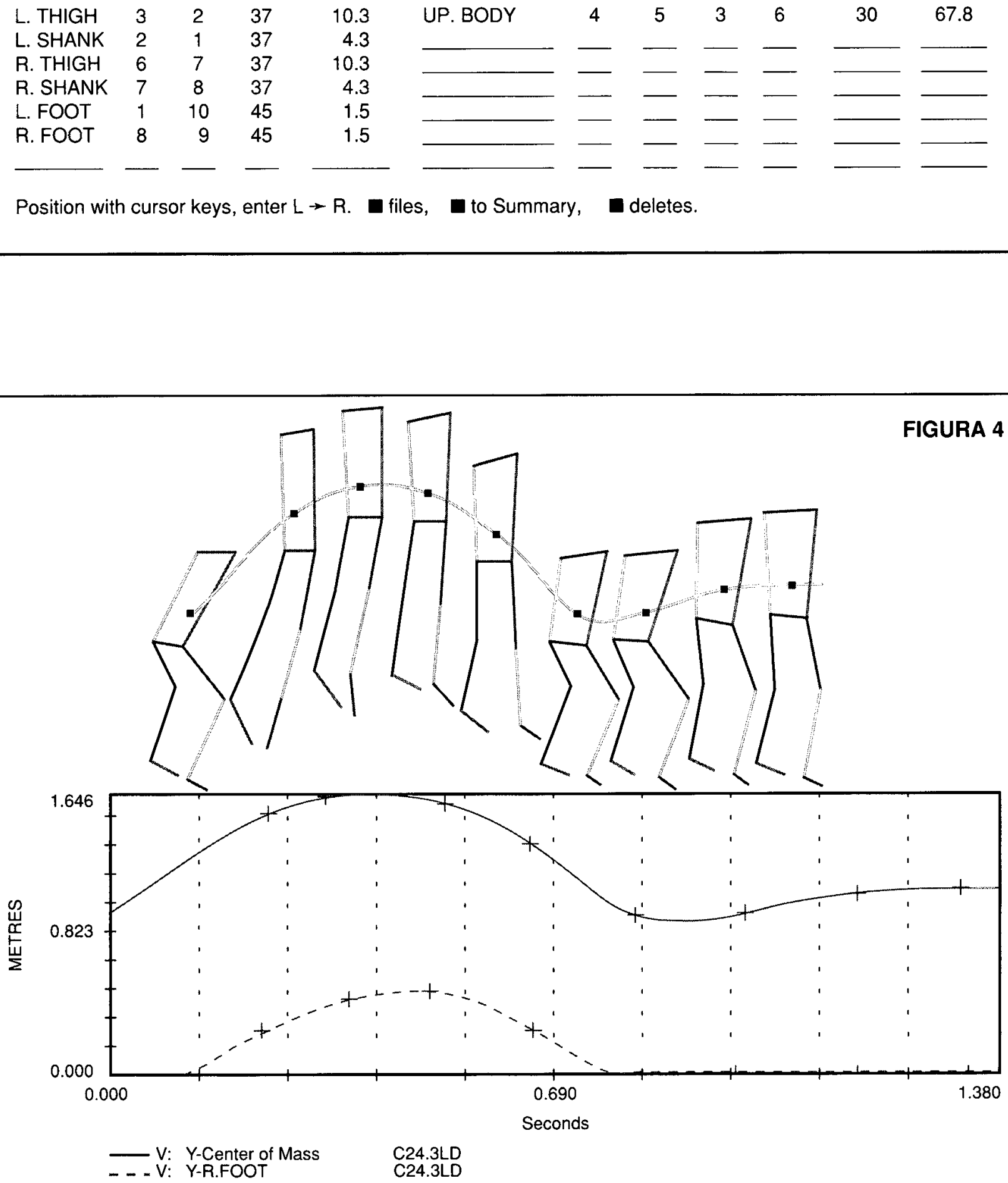


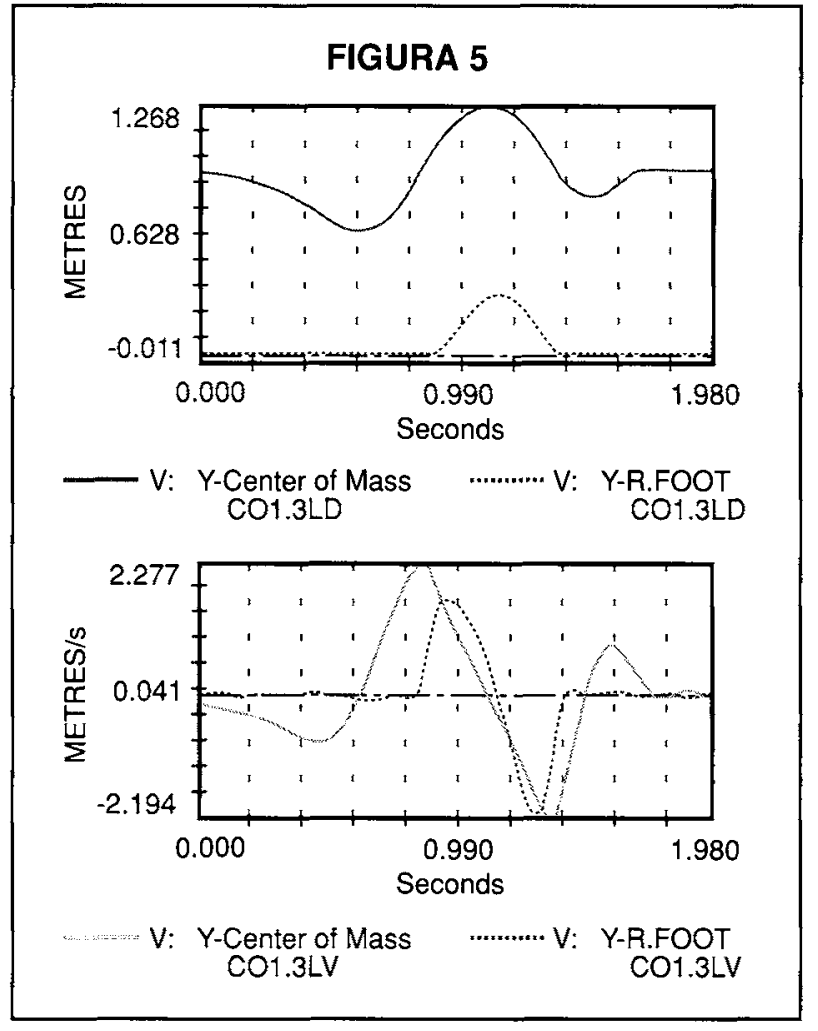

Una vez digitalizadas las imágenes, se presentó un modelo el cual permitió observar la trayectoria del centro de gravedad, así como medir la altura recorrida por el centro de gravedad instantáneo. Se tomaron los siguientes puntos de referencia (fig. 4 y 5 ):

- Instante en que la altura del centro de gravedad es menor.

- Instante inmediatamente anterior al despegue, considerado éste como el momento del despegue del primer dedo del último pie que deja de contactar con el suelo.

- Instante en que la altura del centro instantáneo de gravedad es máxima ( $\mathrm{V}-0)$.

- Instante inmediatamente anterior al aterrizaje, considerado como tal el momento de contacto con la plataforma del primer dedo del primer pie que contacte con ésta.

- Instante en que el centro de gravedad es menor posteriormente al aterrizaje.
Se consideran:

- Tiempo de vuelo: a la diferencia de tiempo entre el momento anterior al despegue y el momento anterior al aterrizaje.

- Altura máxima: a la diferencia de alturas del centro instantáneo de gravedad entre el instante anterior al despegue y la altura máxima.

- Altura del recorrido del centro de gravedad: a la diferencia de alturas del centro instantáneo de gravedad entre el punto donde ésta es mínimo y el punto donde es máxima.

- Altura calculada por el tiempo de vuelo de Bosco (altura de Bosco) dato suministrado por el sistema ergojump.

\section{RESULTADOS}

Se comparó porcentualmente la altura máxima medida por el sistema de digitalización con la altura de Bosco medida por el tapiz.

El resultado se expresó en tanto por ciento respecto a la altura máxima.

Se procedió al análisis estadístico obteniendo las medidas y desviaciones típicas de distribución según el tipo de salto y el sexo de los sujetos (Tabla 2).

Se realizó una distribución normal de frecuencias de la desviación típica de la población general y se observó un patrón uniformemente distribuido desde -1 desviación típica hasta +1,5 desviación típica según gráfico $\mathrm{A}$.

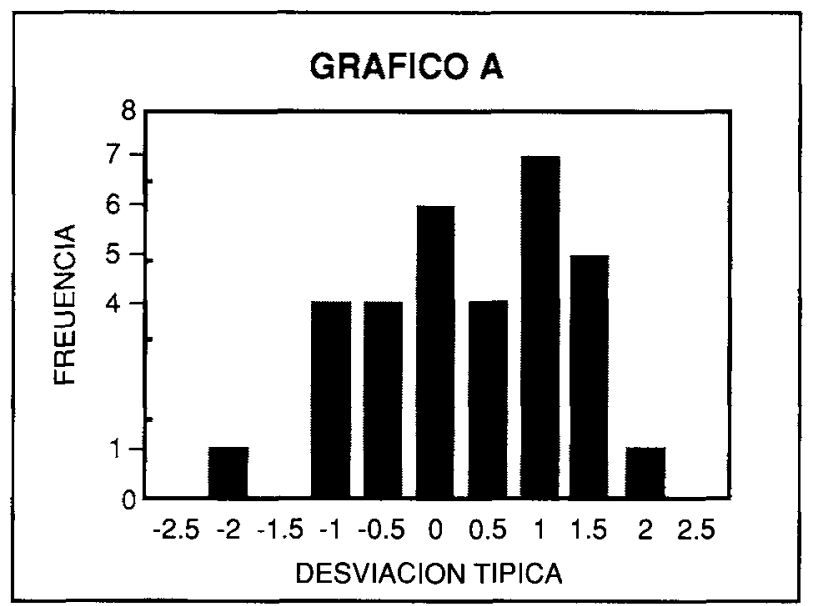

TABLA II

\begin{tabular}{lccccc}
\hline & General & Countermove & Squat Jump & Hombres & Mujeres \\
\hline Número de ensayos & 32 & 16 & 16 & 18 & 14 \\
Media aritmética & 0 & $-0,4$ & 0.6 & 1 & -1 \\
Desviación típica & 5.5 & 4.4 & 6.2 & 4.8 & 6.2 \\
\hline
\end{tabular}




\section{DISCUSION}

El método de medición del instante se despegue del contacto y de nueva toma de contacto adolece de la limitación causada por la frecuencia de filmación del sistema de cámaras, que er de $50 \mathrm{~Hz}$. Sin embargo, al ser el mismo para todos los ensayos, con la sensibilidad de 20 milisegundos, puede ser aceptable, para un tiempo de vuelo de unos 600 milisegundos, un error de un $3 \%$.

El sistema de análisis tridimensional permite establecer la trayectoria según los ejes $\mathrm{X}, \mathrm{Y}, \mathrm{Z}$ del espacio, coniderando el eje $\mathrm{Y}$ como vertical, el eje $\mathrm{X}$ como transversal y el eje $\mathrm{Z}$ como anteroposterior.

El sistema permite medir el desplazamiento estricto en el eje $Y$; los saltos que mostraron un desplazamiento importante en $\mathrm{X}$ o en $\mathrm{Z}$ fueron rechazados.

La posición de partida y la de llegada ha de ser idéntica para no introducir diferencias artificiales en el tiempo total de vuelo sobre el tapiz de Bosco (p. ej. si el saltador encoge las extremidades inferiores, aumenta el tiempo de vuelo falseando el resultado); se rechazaron los saltos con ánguios intersegmentarios de despegue y aterrizaje distintos.

\section{CONCLUSIONES}

1) Del ensayo realizado, se puede inferir que el tapiz de Bosco mide la altura del centro de gravedad del saltador con una desviación de $-5,5 \%$ hasta $18 \%$ respecto a la medición del sistema de análisis del movimiento.

2) Dada la variación de la población observada, se puede deducir la universalidad de la validez del tapiz, ya que la desviación típica es muy similar en todos los grupos.

3) El grado de confianza de un ensayo realizado con el tapiz de Bosco, observando las limitaciones indicadas en la discusión, puede cifrarse en aproximadamente un $90 \%$.

\section{BIBLIOGRAFIA}

1. Bedi, J. «Increase in jumping height associate with maximal effort vertical depth jumps». Res Quart, 1987; 58(1): 11 15.

2. Bosco, C. «Neuromuscula function and mechanical efficiency of human leg extensor muscles during jumping exercises». Acta Physiol Scand, 1982; 114: 543-550.

3. Bosco, C. «A simple method for measurement of mechanical power in jumping». Eur J Appl Physiol, 1983; 50: 273282.

4. Bosco, C. «Stretch-Shortening cycle in skeletal muscle function and physiological considerations on explosive power in man». Atleticastudi, 1985; 16(1): 7-113.

5. Bosco, C. «Valoraciones funcionales de la fuerza dinámi$\mathrm{ca}$, de la fuerza explosiva y de la potencia anaeróbica aláctica con los tests de Boscon. Apunts vol. XXIV. 1987; 151-156.

6. Vitasalo, J. «Evaluation of explosive strength for young and adult athletes». Res Quart, 1987; 59 (1): 9-13. 